\section{Asthmaleitlinien schlecht umgesetzt}

\author{
Die Vorgaben der offiziellen Leitlinien sind auf die Asthmabehand- \\ lung in der Praxis nicht immer direkt übertragbar. Wie groß die \\ Diskrepanz aber tatsächlich ist, überrascht dann doch.
}

D ie Behandlungsmuster bei Asthma bronchiale standen im Fokus einer Studie, für die unselektierte Daten der Kassenärztlichen Vereinigung (KV) Bayerns aus dem Zeitraum April 2005 bis März 2006 ausgewertet wurden. Die Datenbank der KV erfasst über die gesetzlich Krankenversicherten rund 83\% der Bevölkerung Bayerns. Davon litten 4,6\% an Asthma bronchiale, definiert als eine mindestens zweimal bestätigte Diagnose in 2,5 Jahren. Betreut wurden diese Patienten zu 68,3\% vom Hausarzt, zu 8,3\% vom Pneumologen und zu 9,5\% von beiden Disziplinen. Andere gaben als Primärarzt einen Dermatologen, einen
HNO-Arzt oder sogar einen Gynäkologen an (14\%).

Von insgesamt 483.051 Patienten mit Asthma erhielten im Indexzeitraum nur $61,4 \%$ eine Pharmakotherapie. Die Behandlungspersistenz als wesentlicher Aspekt einer erfolgreichen Therapie schätzten die Autoren der Studie über die angenommene mittlere Tagesdosis ab. Die mit einer Langzeitmedikation wie inhalativen Glukokortikoiden (ICS) und/oder lang wirksamen $\beta$-Agonisten (LABA) behandelten Patienten machten nur $65,1 \%$ aus (ICS: 25,1\%; ICS und LABA: 35,4\%; LABA: 4,7\%). Über ein Jahr hinweg erhielt die Mehrzahl von ih- nen $(58,1 \%)$ nicht mehr als 90 Tagesdosen, und nur etwa jeder hundertste war durchgehend versorgt. Häufiger wurden kurz wirksame $\beta$-Agonisten angewendet $(65,6 \%) .6,2 \%$ der damit behandelten Patienten erhielten sogar mehr als 360 Tagesdosen.

Fazit: Die Diskrepanz zwischen offiziellen Empfehlungen und der klinischen Praxis ist enorm. Insgesamt wird nur gut die Hälfte der Patienten mit Asthma bronchiale den Leitlinien entsprechend therapiert. Zu wenige Erkrankte erhalten eine adäquate Langzeittherapie, und inhalative Glukokortikoide sind dabei unterrepräsentiert.

Hasford J et al. Persistence with asthma treatment is low in Germany especially for controller medication - a population based study of 483051 patients. Allergy 2010; 65 : $347-54$

\title{
Asthma-Sprays mit Umfeldwirkung
}

\section{Inhalative Glukokortikoide verursachen bei ihren Anwendern selten eine Sensibilisierung. Vermutlich sind die Patienten selbst durch eine orale Toleranz geschützt. Aber wie steht es mit ihrem passiv expo- nierten Umfeld?}

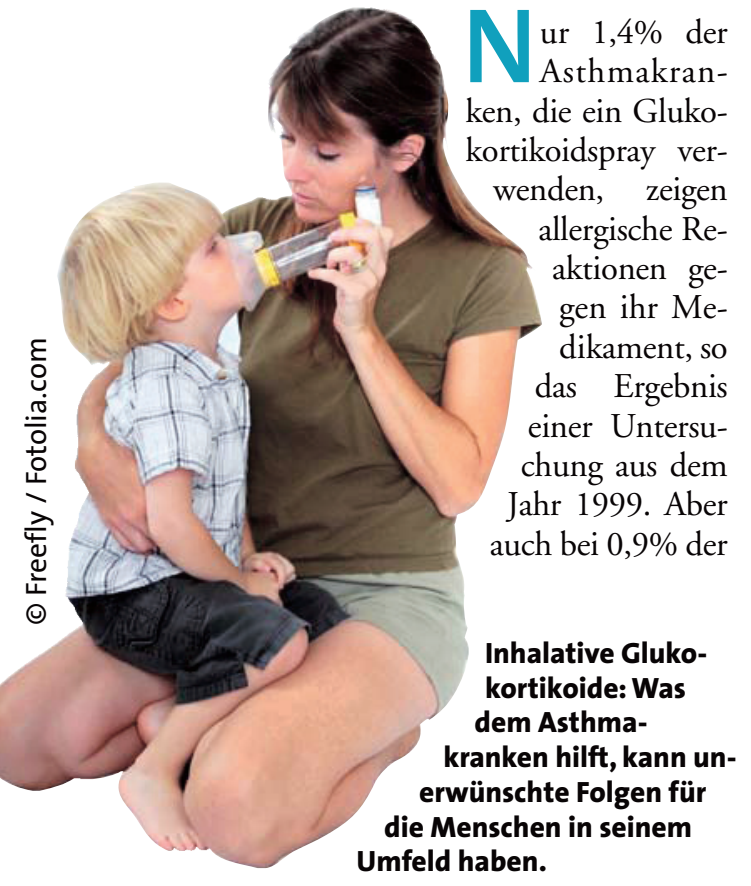

Nichtasthmatiker fand sich eine Glukokortikoid-Sensibilisierung. Für deren Ursachen interessierten sich jetzt zwei belgische Dermatologinnen. Retrospektiv werteten sie die Patchtestergebnisse von Patienten der Universitätsklinik in Löwen aus.

In einem Zeitraum von 18 Jahren hatten 315 Personen positiv auf Glukokortikoide wie Tixocortol, Budesonid, Hydrocortison oder Prednison reagiert. 15 der Personen (5\%) verwendeten selbst keine Glukokortikoide, betreuten oder lebten aber mit Patienten zusammen, die regelmäßig Glukokortikoide inhalierten. Sie wurden offenbar durch passive Inhalation bzw. Hautkontakt sensibilisiert. Ein Drittel der Personen war ausschließlich gegenüber Budesonid sensibilisiert. Neun Personen zeigten klinische Symptome einer durch Aeroallergene vermittelten Kontaktderma- titis. Bei einer Frau trat zum Beispiel eine Kontaktdermatitis im Gesicht auf, nachdem sie ihrem Kind bei der Glukokortikoidinhalation geholfen hatte. Eine andere Frau war in einer Kindertagesstätte angestellt und berichtete über einen Rückgang ihrer KontaktdermatitisSymptome während des Urlaubs. Auch sie unterstützte die Kinder regelmäßig bei der Inhalation.

Die 15 Personen wurden 2009 nochmals kontaktiert, zehn von ihnen (66\%) antworteten. Von sieben Personen mit klinischen Symptomen waren vier weiterhin gegenüber Glukokortikoiden exponiert, sie mieden aber Kontaktsituationen und berichteten über eine deutliche Besserung.

Fazit: Eine passive, über die Luft vermittelte Glukokortikoidexposition kann vereinzelt zu einer primären Sensibilisierung bis hin zur Kontaktdermatitis führen. Betroffen sind häufig Betreuer von asthmakranken Kindern, die diesen bei der Inhalation helfen.

Baeck $M$ et al. Patients with airborne sensitization/contact dermatitis from budesonide-containing aerosols, by proxy'. Contact Dermatitis 2009; 61: 1-8 